\title{
Brief Report: Insight into Illness and Social Attributional Style in Asperger's Syndrome
}

\author{
Nyaz Didehbani • Mujeeb U. Shad • Michelle R. Kandalaft • \\ Tandra T. Allen - Carol A. Tamminga - Daniel C. Krawczyk · \\ Sandra B. Chapman
}

Published online: 24 April 2012

(C) Springer Science+Business Media, LLC 2012

\begin{abstract}
A number of psychiatric illnesses have been recognized to have some level of insight deficits, including developmental disorders, such as Asperger's Syndrome (ASP). However insight into illness has not been empirically investigated in ASP and little research has examined how individuals with ASP view their deficits. This is the first study to assess insight and the relationship between insight and externalizing bias (EB) in ASP. Participants with ASP $(n=21)$ and healthy controls $(n=24)$ were recruited. Attributional style was assessed with the internal, personal, and situational attribution questionnaire. Insight was assessed with both a clinician-administered and a selfadministered measure. Results revealed that EB was negatively correlated with insight as assessed with the clinician-administered but not the self-administered measure of insight.
\end{abstract}

Keywords Asperger's · Autism · Insight ·

Social cognition $\cdot$ Attribution, externalizing bias

N. Didehbani $(\bowtie) \cdot$ M. R. Kandalaft · T. T. Allen ·

D. C. Krawczyk · S. B. Chapman

Center for BrainHealth ${ }^{\circledR}$, The University of Texas at Dallas, 2200 W. Mockingbird Lane, Dallas, TX 75235, USA

e-mail: nyaz.didehbani@UTDallas.edu

M. U. Shad

Department of Psychiatry, University of Texas Medical School at Houston, Houston, TX, USA

C. A. Tamminga - D. C. Krawczyk

Department of Psychiatry, University of Texas Southwestern Medical Center at Dallas, Dallas, TX, USA

\section{Introduction}

Insight into illness is a widely recognized multidimensional concept that encompasses awareness of illness, its symptoms, need for treatment, and the social consequences of illness (Amador et al. 1994). A number of psychiatric illnesses have some level of insight deficits, with the majority of research focusing on the impact of poor insight in schizophrenia (SCZ) (Amador et al. 1994, 1993; Quee et al. 2011). Like SCZ, individuals with Asperger's Syndrome (ASP) demonstrate poor social awareness (Johnson et al. 2009), but to the author's knowledge, no one has empirically investigated insight into illness in ASP and thus little is known about how ASP view their deficits.

Awareness and insight are two related but distinguishable concepts. Making a distinction between the two is informative because while most of the research in autism focuses on self-awareness, few if any efforts are directed toward elucidating insight into illness. Markova and Berrios (2011) argue that the constructs of "awareness" and "insight" are separable, thus requiring different methods of investigation. They define awareness as a direct measurement of the symptom or impairment, whereas insight is an extension of awareness and incorporates how an individual makes sense of the symptom or impairment. Therefore, insight, the focus of this paper, is a more complex phenomenon than awareness and requires an individual to interpret their symptoms based on prior experiences and self-knowledge.

Insight into illness is a difficult construct to measure as it is based on individuals' self-perception of their symptoms. This construct is closely tied to the positive illusory bias, which is defined as the difference between a person's self-perception of their abilities and their actual abilities (Owens et al. 2007). Studies investigating children with 
Attention Deficit/Hyperactivity Disorder (ADHD) have shown that they tend to overestimate their abilities and may not be aware of some of their limitations. Some researchers have suggested that this lack of awareness may serve to protect their self value without harming their self-confidence (Hoza et al. 2004; Owens et al. 2007). Likewise, insight into illness for diseased populations is an important measure of their own understanding of their illness. While individuals with limited awareness into illness may be protecting their self-esteem, poor insight may contribute to poorer treatment outcomes as commonly reported in schizophrenia (Schwartz et al. 1997).

Unfortunately, little is known about insight into illness in ASP even though it has been clinically noted that individuals with ASP often lack insight into their own social and cognitive deficits (Carrington et al. 2003). Some case studies have related poor insight with attributional style and proposed that individuals with ASP may demonstrate a stronger cognitive bias in assigning blame to others due to the difficulty in recognizing personal roles in social interactions (Berney 2004; Hare et al. 1999). Lombardo and Baron-Cohen (2011) have further acknowledged that selfawareness is necessary for understanding others roles and perspectives. It is thus important to empirically evaluate how individuals with ASP view both their roles and others in social situations (e.g. social attribution).

Research in causal attribution with Autism Spectrum Disorders (ASD) has focused on moral reasoning and making moral judgments, such as attributing blame or forgiveness. Overall, researchers explain that individuals with ASD are able to make basic moral judgments (Leslie et al. 2006) and understand social rules (Takeda et al. 2007) but have difficulty incorporating intent and motives in explaining moral decisions (Grant et al. 2005; Roge and Mullet 2011). For this reason it is beneficial to examine attributional style and how ASP view individual roles in social situations as provided in the Internal, Personal, and Situational Attribution Questionnaire (IPSAQ; Kinderman and Bentall 1996). The IPSAQ provides a social situation such as, "A friend refused to talk to you" and asks if this situation happened because of you, the other person, or the situation. The questions on the IPSAQ force the individual to use self-knowledge and prior experiences to make a social judgment. It assesses how individuals view their role versus others in a social context. Overall, it provides a standardized methodology to examine social attribution and cognitive biases such as externalizing bias (assigning blame to another person for a negative event).

No research to date has investigated a relationship between insight into illness and social attribution in individuals with ASP and only few have investigated social attribution bias (e.g., externalizing bias) in ASP. The current preliminary study takes a novel approach to illuminate insight by comparing the relationship between insight into illness and externalizing bias (EB), a self-serving bias within a social situation in individuals with ASP. Earlier literature in SCZ has modeled EB as a defense mechanism to maintain self-esteem through misattribution of negative self-concepts thereby contributing to poor insight (Amador et al. 1993). Moreover, misattribution in the form of overestimating abilities (positive illusory bias) may serve as coping mechanism in some clinical populations (Hoza et al. 2004). Although one pathology in ASP is impaired social awareness, individuals with ASP may also exhibit a tendency to use EB in blaming others for their own deficits in social situations. As a result, we wanted to test if individuals with ASP demonstrated different social attributional styles compared to healthy controls and also examine the relationship between these constructs in ASP. We first examined differences on the IPSAQ between ASP and agematched controls and predicted that controls would demonstrate a higher EB than indivudals with ASP. We next examined the relationship between measures of insight and social attribution and predicted that scores on social EB and level of insight would be inversely related. Therefore, individuals with ASP with poor insight may demonstrate a high social EB (blame others more than self for negative situations) to protect self-worth as established in SCZ. A similar relationship between self-worth and self-attributions for negative events was found in children with ADHD; children with high self-worth, tend to blame others for negative events (Hoza et al. 1993), indicative of a high EB. We also wanted to explore if the hypothesized relationship between social EB and insight would be impacted if insight was assessed with more than just an existing selfreport insight measure, Birchwood Insight Scale (BIS; Birchwood et al. 1994), because research suggests individuals with ASP do not always rate deficits accurately on self-report measures (Green et al. 2000; Koning and Magill-Evans 2001; Russell and Sofronoff 2005). As a result we selected the widely used clinician-administered Scale to Assess Unawareness of Mental Disorder-Abbreviated Version (SUMD-AV; Amador et al. 1993). Finally, an exploratory analysis examined the relationship between Autism Diagnostic Observation Schedule (ADOS), social attribution, and insight.

\section{Methods}

Participants

Twenty-one individuals with ASP and 24 controls were recruited for the study. The participants with ASP included 14 males and 7 females with ages ranging from 18 to 34 $(M=23.05, \quad \mathrm{SD}=4.88)$. The healthy control group 
included 13 males and 11 females with ages ranging from 18 to $39(M=25.71, \mathrm{SD}=5.55)$.

Participants with ASP and healthy controls were recruited at the Center for BrainHealth, University of Texas at Dallas (UTD). The clinical group had a current primary diagnosis of ASP as defined by the Diagnostic and Statistical Manual of Mental Disorders, 4th ed. (DSMIV; APA 2000) criteria. The diagnosis for the participants with ASP was confirmed by the Autism Diagnostic Observation Schedule (ADOS; Lord et al. 2000) by trained clinicians involved with the study. Participants were excluded if their clinical status required inpatient treatment, if they had a history of seizures, if they reported substance dependence within the last 3 months, or if they had an estimated IQ less than 80 as measured by the WASI. The IQ cutoff was set at 80 , as this investigation was part of a larger intervention study in which participants were required to have at least this IQ. Participants who met all inclusion and failed to meet exclusion criteria were enrolled in the study. All individuals provided written informed consent to participate and all procedures were approved by and carried out in accordance with the UTD Institutional Review Boards.

\section{Measures}

\section{Autism Diagnostic Observation Schedule (ADOS;} Lord et al. 2002)

The ADOS is a semi-structured assessment used for individuals suspected to have autism and consists of various activities that allow the examiner to observe social and communication behaviors. It includes four modules depending on his or her expressive language level and chronological age. Only Module 4 (for fluent adolescents and adults) was administered and scored by trained raters for the current study. The administration of the ADOS module 4 consisted of all standard activities including the optional daily living items in order to gather a detailed history.

\section{Wechsler Abbreviated Scale of Intelligence (WASI; Wechsler 1999)}

We calculated an estimated IQ from two WASI subtests: Vocabulary and Matrix Reasoning which has an average reliability coefficient of .96 .

\section{Internal Personal Situational Attributions Questionnaire (IPSAQ; Kinderman and Bentall 1996)}

This measure assessed how individuals attribute causes to social events (16 negative and 16 positive). Participants attributed the cause of a friend's behavior (e.g., "a friend thinks you are trustworthy") to themself (internal attribution), another person (personal attribution), or an outside factor (situational attribution). Three subscales were derived from the 16 positive items by adding the number of internal, personal, or situational attributions for the positive situations and 3 subscales are derived from the 16 negative items by adding the number of internal, personal, or situational attributions for the negative situations. Two social attribution bias scores were calculated from the subscales: an externalizing bias (EB) and a personalizing bias (PB). The EB was calculated by subtracting the total number of internal attributions for the negative items from the total number of internal attributions for the positive items. Thus, positive EB scores indicated a self-serving bias whereas negative scores indicate a tendency to blame oneself more for negative events than positive events. PB was calculated by dividing the number of personal attributions by the sum of the personal and situational attributions for either negative situations ( $\mathrm{PB}$ negative) or positive situations (PB positive). A PB score of less than .5 indicated a tendency to blame the situation rather than the person for negative events. Both the EB and PB demonstrate acceptable internal reliability with a Cronbach's alpha of .72 and .76 respectively (Kinderman and Bentall 1996). In the current study, the Cronbach alpha coefficient was .72.

\section{Birchwood Insight Scale (BIS; Birchwood et al. 1994)}

This self-report scale was comprised of 8 items that measure different aspects of insight into illness such as awareness of symptoms, illness, and the need for treatment. The individual rated each item by choosing "agree," "unsure," or "disagree" for each statement. Each item is scored as 0,3 , or 5 and then summed for a total score. Low scores indicated poor insight. The BIS has acceptable internal reliability with a Cronbach's alpha of .75 (Birchwood et al. 1994; Sapara et al. 2007). The current study utilized a slightly modified version of the BIS to administer to participants with ASP. The BIS assessed an individual's level of insight into their mental illness but since individuals with ASP in our sample tended not to view their diagnoses as a mental disorder, the words "mental illness" were replaced with "my diagnoses." This observation was based on our pilot testing of the original version of the BIS whereby most participants with ASP needed clarification for "mental disorder" and had difficulty answering the questions. Additionally, for each question on the BIS, the clinician clarified "my diagnosis" as the person's diagnosis of ASP. In the current study, it was verbally confirmed by each participant that he/she understood the meaning of "my diagnoses" as having ASP. The Cronbach alpha coefficient in this study was .32 . 
Scale to Assess Unawareness of Mental DisorderAbbreviated Version (SUMD-AV; Amador et al. 1993)

An abbreviated version of this semi-structured clinician administered interview was utilized in this study. This abbreviated version was comprised of three general items: (1) Awareness of having a mental disorder; (2) Awareness of the need for treatment; and, (3) Awareness of the social consequences of the illness. For example, the clinician asked "do you believe that your problems with social situations are due to your diagnosis," and the clinician then proceeded to ask follow-up questions to clarify. Trained clinicians then used a 5-point likert scale to rate the participant's level of insight into illness based on the participant's response and summed each item for a total score. These same clinicians also administered the ADOS and were able to use their best clinical judgment in assessing level of insight. High scores indicated poor insight. In the current study, the Cronbach alpha coefficient was .64.

\section{Procedures}

All participants completed written informed consent, WASI, and IPSAQ. Only individuals with ASP completed the two insight measures.

\section{Statistical Analysis}

Statistical analyses were conducted using Statistical Package for Social Sciences (SPSS) version 18.0. Means and standard deviations were calculated for the demographic variables (age and education), estimated IQ, IPSAQ scores, and the two insight measures (SUMD-AV and BIS). Next, one-way analysis of variance (ANOVA) was used to compare ASP with controls across the IPSAQ. Next, correlations were run to investigate the relationship of scores on insight measures with the IPSAQ. Finally, an exploratory correlation between the ADOS subtests with insight into illness and social attribution was run. Some data violated the assumption of normality and therefore nonparametric Spearman's correlations were used.

\section{Results}

A summary of demographic information across both groups is presented in Table 1. The ANOVA revealed that the groups did not differ in education, age, or estimated FSIQ using the WASI.

A summary of the insight, IPSAQ, and ADOS scores are presented in Table 2. Based on an ANOVA, no significant
Table 1 Summary of demographic information

\begin{tabular}{lccc}
\hline & $\begin{array}{l}\text { Asperger's } \\
\text { syndrome }\end{array}$ & Control & $p$ values \\
\hline Age (years) & $23.05(4.83)$ & $25.81(5.89)$ & .10 \\
Education (years) & $13.24(1.70)$ & $13.73(1.56)$ & .11 \\
Estimated FSIQ & $112.33(10.59)$ & $113.14(11.17)$ & .54 \\
$\quad$ standard score) & & & \\
Vocabulary (t-score) & $56.00(9.98)$ & $58.21(8.35)$ & .43 \\
Matrix Reasoning (t- & $58.00(4.61)$ & $58.00(7.08)$ & 1.00 \\
$\quad$ score) & & & \\
\hline$p<.05^{*}$ & & &
\end{tabular}

Table 2 Means and standard deviations of all measures across groups

\begin{tabular}{|c|c|c|c|}
\hline Measures & $\begin{array}{l}\text { Asperger's } \\
\text { syndrome }\end{array}$ & Control & $p$ values \\
\hline $\begin{array}{l}\text { IPSAQ: Positive internal } \\
\text { attribution }\end{array}$ & $8.53(3.36)$ & $9.16(3.24)$ & .59 \\
\hline $\begin{array}{l}\text { IPSAQ: Positive personal } \\
\text { attribution }\end{array}$ & $3.87(3.20)$ & $3.16(2.19)$ & .48 \\
\hline $\begin{array}{l}\text { IPSAQ: Positive situational } \\
\text { attribution }\end{array}$ & $3.60(2.44)$ & $3.68(2.73)$ & .93 \\
\hline $\begin{array}{l}\text { IPSAQ: Negative internal } \\
\text { attribution }\end{array}$ & $7.13(4.07)$ & $4.89(2.64)$ & .06 \\
\hline $\begin{array}{l}\text { IPSAQ: Negative personal } \\
\text { attribution }\end{array}$ & $5.67(3.87)$ & $6.53(1.78)$ & .39 \\
\hline $\begin{array}{l}\text { IPSAQ: Negative } \\
\text { situational attribution }\end{array}$ & $3.20(2.07)$ & $4.58(2.85)$ & .13 \\
\hline IPSAQ: Externalizing bias & $1.62(5.26)$ & $4.13(3.92)$ & .08 \\
\hline $\begin{array}{l}\text { IPSAQ: Personalizing bias } \\
\text { (negative events) }\end{array}$ & $.58(.23)$ & $.59(.21)$ & .86 \\
\hline $\begin{array}{l}\text { IPSAQ: Personalizing bias } \\
\text { (positive events) }\end{array}$ & $.45(.37)$ & $.47(.26)$ & .83 \\
\hline $\begin{array}{l}\text { Birchwood insight scale } \\
\text { total }(0-12)\end{array}$ & $5.86(2.19)$ & N/A & N/A \\
\hline SUMD-AV & $4.71(1.74)$ & N/A & N/A \\
\hline ADOS communication total & $3.60(1.73)$ & N/A & N/A \\
\hline $\begin{array}{l}\text { ADOS reciprocal social } \\
\text { interaction total }\end{array}$ & $5.60(2.26)$ & N/A & N/A \\
\hline $\begin{array}{l}\text { ADOS } \\
\text { communication + social } \\
\text { interaction }\end{array}$ & $9.2(3.56)$ & N/A & N/A \\
\hline $\begin{array}{l}\text { ADOS imagination/ } \\
\text { creativity }\end{array}$ & $.89(.74)$ & N/A & N/A \\
\hline $\begin{array}{l}\text { ADOS stereotyped } \\
\text { behavior total }\end{array}$ & $2.00(1.03)$ & N/A & N/A \\
\hline
\end{tabular}

IPSAQ internal, personal, situational attributions questionnaire, $S U M D-A V$ scale to assess unawareness of mental disorder-abbreviated-version, $A D O S$ Autism diagnostic observation schedule $p<.05^{*}$

differences were found between the ASP and control groups on the six IPSAQ subscales or the two cognitive biases. 
Based on Spearman's correlation coefficients, social EB as measured by the IPSAQ was negatively correlated with insight as assessed with the clinician-administered SUMD$\mathrm{AV}$ in patients with ASP (rho $=.64, p=.002$ ). In looking at individual subscale scores on the IPSAQ, insight as assessed with SUMD-AV was significantly correlated with negative internal attribution (rho $=-.66, p=.007$ ) and negative personal attribution (rho $=.54, p=.04$ ). There were no significant correlations between the IPSAQ scores and insight as assessed with the self-administered BISMOD. The two insight measures, SUMD-AV and BIS were also not significantly correlated with one another.

Finally, an exploratory analysis investigated the relationship between the ADOS subtests with insight into illness and social attribution. Spearman's correlational analyses revealed a significant correlation between the ADOS Reciprocal Social Interaction subscale with the BIS Treatment subscale (rho $=.47, p=.04$ ) and the ADOS Communication subscale with the BIS Symptom subscale (rho $=-.49, p=.03$ ).

\section{Discussion}

The aim of this study was to compare social attributional style in relation to insight into illness in individuals with ASP. We found no significant group differences in social EB or PB between ASP and control groups. However, both groups obtained positive EB scores, indicating a selfserving bias. This finding indicated that social attributional bias may not only be associated with psychopathology but healthy controls may also have a greater tendency to use personal rather than situational external attributions for negative events (Taylor and Brown 1988). Taylor and Brown (1988) described these adaptive misconceptions as "illusions" which enable a person to engage in productive work. Positive illusions may promote positive mood and may enhance cognitive abilities such as efficient use of problem-solving and decision-making skills (Taylor and Brown). Researchers have also shown that healthy adults tend to inflate their abilities and evaluate themselves more favorably compared to others, i.e., positive illusory bias (Owens et al. 2007), which may again have a positive impact on cognitive functioning and self-esteem (Taylor and Brown).

Individuals with ASP showed a significant correlation between EB on the IPSAQ and level of insight, but only when insight was assessed with the clinician-administered insight measure, SUMD-AV. Consequently, as ASP become more aware of their deficits related to their diagnosis, they may believe that they are at fault for negative social consequences which may contribute to a lowered self-esteem. Therefore, as individuals with ASP start recognizing shortcomings, they may become more aware and sensitive to the consequences of their deficits. This is not surprising, because researchers have found that as individuals with SCZ gain more insight into their illness, they may become more depressed (Cotton et al. 2012; Drake et al. 2004; Saeedi et al. 2007) and tend to have lower self-esteem (Drake et al.). In looking at the subscale scores on the IPSAQ, ASP with high insight were more likely to blame themselves for negative social events (negative internal) and less likely to blame others for negative social events (negative personal).

In comparing the two insight measures, it is possible that higher scores on clinician-assessed insight scale may have been a result of the specific questions used on the abbreviated SUMD-AV which contained more general questions about the ASP diagnoses. The SUMD-AV administration enables individuals to explain how they feel about their diagnoses, previous treatment, and social deficits. The SUMD-AV was also designed to address insight across major psychiatric disorders. The self-report BIS may have been too specific and too symptom-based for individuals with ASP despite our modification. It is also possible that some individuals with ASP do not exhibit certain symptoms and for this reason may appear to lack insight into the illness. Therefore, the lower scores may not necessarily indicate lack of insight but rather a lack of symptoms. This may help explain why EB was only correlated with SUMD-AV and not BIS. Previous studies have noted that individuals with ASP often do not accurately rate deficits in self-reports (Green et al. 2000; Koning and Magill-Evans 2001; Russell and Sofronoff 2005). However, there is no self-report or clinician-administered measure of insight designed specifically for individuals with ASP despite the limited self and social awareness commonly cited in this population.

Of note, we did not find any relationship between the two insight measures. This may again be a result of the nature of each measure. The self-reported BIS was designed specifically for individuals with SCZ whereas the clinician-rated SUMD-AV was designed for use across all psychiatric disorders. These two insight measures may have also captured different psychological underpinnings of insight deficits or may have assessed different degrees of awareness of symptoms versus insight into illness. Again, Markova and Berrios (2011) argue that "awareness" and "insight" are different constructs and often used interchangeably. Consequently, it is possible that in using the SUMD-AV the clinician made a judgment about the participants' insight into illness in relation to their functioning. The SUMD-AV allowed the clinician to account for some level of severity and acknowledge individual differences in the scoring based on each participant's response and explanation of their deficits. The BIS on the other hand, 
may have focused more on the participant's awareness of their symptoms, illness, and treatment. Additionally, the BIS scoring may not fit as well with this population, because higher scores indicate that the individual is endorsing more problems and thus appears to have more insight. It does not account for symptom severity and individual differences. Therefore specific symptoms listed on the BIS may not be relevant for a given individual and the person will thus not endorse it. For example, one of the statements on the BIS asked if the participant feels the need to be seen by a mental health provider which some of the participants responded that they do not think they need psychological care. For some individuals, this is a valid response and does not indicate poor insight. As a result, the low insight scores for these less symptomatic individuals will indicate poor insight which is not necessarily the case. These differences in administration (clinician versus self) and quality of scoring may help explain why social EB was related to insight as measured by the SUMD-AV and not the BIS.

In examining the ADOS subscales with measures of insight into illness and social attribution, two relationships with the BIS were observed. The Reciprocal Social Interaction subscale on the ADOS was significantly correlated with the BIS Treatment subscale. This suggested that a person who acknowledged a higher need for treatment may be less effective in reciprocal social interaction. However, these results should be interrupted with caution because many of the participants in the current study did not endorse a need for treatment for their ASP diagnosis as measured on the BIS. There was also a significant relationship between the Communication subscale on the ADOS with the BIS Symptom subscale. This finding indicated that individuals with high insight into symptoms related to their ASP diagnosis, were able to communicate more effectively in a social interaction. It is important to note that the overall mean for the Communication subscale on the ADOS was the only score to reach the Autism cutoff. The other subscales only met the Autism Spectrum cutoff. The higher score on the Communication subscale indicated a more severe range of functioning for this domain. There were no other significant relationships with the ADOS and one reason that the BIS was the only measure to correlate may again be related to the idea that the BIS is a measure of symptomology much like the ADOS. However, because of the limitations of the BIS, it is difficult to fully interpret these findings.

Overall these results indicate that insight into illness is related to social attribution for individuals with ASP. There was a strong relationship between measures of insight as assessed with the clinician administered SUMD-AV and social EB as assessed with the IPSAQ. In looking more closely at individual IPSAQ subscales, insight was related to how individuals with ASP attribute negative events either to themselves (internal) or to others (personal). Further investigation into the subscales in comparison to controls would provide more specific information in regards to attributional style. Other limitations include the use of an abbreviated clinician administered scale to assess insight and the use of an insight scale directed toward individuals with SCZ. The insight measures were also limited by their low internal reliability as calculated in this study. Additionally, the SUMD was rated only by one trained clinician which may have biased the scores. Future use of this measure may benefit from having two raters that reach consensus over ratings Therefore, the findings from this study will need to be replicated in a larger sample size using a more comprehensive scale for insight assessment than the one used in this study. The current study was also limited in that insight deficits in individuals with ASP were not assessed by other informant raters such as close family members or friends. An insight into illness measure that addresses global concerns from multiple informants would provide a more comprehensive profile of insight deficits. In addition, the observation that different insight measures may assess different psychological mechanisms, suggests that an insight measure that directly assesses a person's understanding of how the symptoms relate to social functioning may be more useful in comprehensively assessing insight deficits. Finally, future research should assess selfesteem and its relationship with insight into illness and social attribution. Research in ADHD and SCZ have shown that self-esteem plays a role in social attribution and an investigation of the interactions between insight into illness, social attribution, and self-esteem seems warranted.

Despite these limitations, this study is the first to examine and explore the relationship between social attributional styles and insight into illness in ASP. These findings fill a void in the ASP literature by providing an empirical research study which investigated insight into illness deficits in this clinical group known to lack social awareness. Further investigation into the effects or contributing factors associated with poor insight, will help researchers and clinicians design targeted interventions for individuals with ASP.

Acknowledgments This work was supported by funding from the Lattner Family Foundation, the John A. and Lee Wacker Social Cognition Project, and Crystal Charity Ball.

\section{References}

Amador, X. F., Flaum, M., Andreasen, N. C., Strauss, D. H., Yale, S. A., Clark, S. C., et al. (1994). Awareness of illness in schizophrenia and schizoaffective and mood disorders. Archives General Psychiatry, 51(10), 826-836. 
Amador, X. F., Strauss, D. H., Yale, S. A., Flaum, M. M., Endicott, J., \& Gorman, J. M. (1993). Assessment of insight in psychosis. American Journal of Psychiatry, 150(6), 873-879.

APA. (2000). Diagnostic and statistical manual of mental disorders, fourth edition, text revision. Washington, DC: American Psychiatric Association.

Berney, T. (2004). Asperger syndrome from childhood to adulthood. Advances in Psychiatric Treatment, 10, 341-351.

Birchwood, M., Smith, J., Drury, V., Healy, J., Macmillan, F., \& Slade, M. (1994). A self-report insight scale for psychosis: Reliability, validity, and sensitivity to change. Acta Psychiatrica Scandinavica, 89, 62-67.

Carrington, S., Templeton, E., \& Papinczak, T. (2003). Adolescents with Asperger syndrome and perceptions of friendship. Focus on Autism \& Other Developmental Disabilities, 18(4), 211-218.

Cotton, S. M., Lambert, M., Schimmelmann, B. G., Mackinnon, A., Gleeson, J. F. M., Berk, M., et al. (2012). Depressive symptoms in first episode schizophrenia spectrum disorder. Schizophrenia Research, 134(1), 20-26. doi:10.1016/j.schres.2011.08.018.

Drake, R. J., Pickles, A., Bentall, R. P., Kinderman, P., Haddock, G., Tarrier, N., et al. (2004). The evolution of insight, paranoia and depression during early schizophrenia. Psychological Medicine, 34, 258-292. doi:10.1017/S0033291703008821.

Grant, C. M., Boucher, J., Riggs, K. J., \& Grayson, A. (2005). Moral understanding in children with autism. Autism, 9(3), 317-331. doi: $10.1177 / 1362361305055418$.

Green, J., Gilchrist, A., Burton, D., \& Cox, A. (2000). Social and psychiatric functioning in adolescents with Asperger syndrome compared with conduct disorder. Journal of Autism and Developmental Disorders, 30(4), 279-293.

Hare, D. J., Jones, J. P. R., \& Paine, C. (1999). Approaching realilty: The use of personal construct assessment in working with people with Asperger syndreom. Autism, 3, 165-176.

Hoza, B., Gerdes, A. C., Arnold, L. E., Molina, B. S. G., Epstein, J. N., Hechtman, L., et al. (2004). Self-perceptions of competence in children with ADHD and comparison children. Journal of Consulting and Clincial Psychology, 72(3), 382-391. doi: 10.1037/0022-006X.72.3.382.

Hoza, B., Pelham, W. E., Milich, R., Pillow, D., \& McBride, K. (1993). The self-percpetions and attributions of Attention Deficit Hyperactivity Disordered and nonreferred boys. Journal of Abnormal Child Psychology, 21(3), 271-286. doi:0091-0627/ 93/0600-0271.

Johnson, S. A., Filliter, J. H., \& Murphy, R. R. (2009). Discrepancies between self- and parent-perceptions of autistic traits and empathy in high functioning children and adolescents on the autism spectrum. Journal of Autism and Developmental Disorders, 39(12), 1706-1714. doi:10.1007/s10803-009-0809-1.

Kinderman, P., \& Bentall, R. P. (1996). A new measure of causal locus: The internal, personal and situational attributions questionnaire. Personality and Individual Differences, 20(2), 261-264

Koning, C., \& Magill-Evans, J. (2001). Social and language skills in adolescent boys with Asperger syndrome. Autism, 5(1), 23-36.
Leslie, A. M., Mallon, R., \& DiCorcia, J. A. (2006). Transgressors, victims, and cry babies: Is basic moral judgement spared in autism? Social Neuroscience, 1(3-4), 270-283. doi:10.1080/ 17470910600992197.

Lombardo, M. V., \& Baron-Cohen, S. (2011). The role of the self in mindblindness in autism. Consciousness and Cognition, 20(1), 130-140. doi:10.1016/j.concog.2010.09.006.

Lord, C., Risi, S., Lambrecht, L., Cook, E. H., Jr, Leventhal, B. L., DiLavore, P. C., et al. (2000). The autism diagnostic observation schedule-generic: A standard measure of social and communication deficits associated with the spectrum of autism. Journal of Autism Developmental Disorders, 30(3), 205-223.

Lord, C., Rutter, M., DiLavore, P. C., \& Risi, S. (2002). Autism diagnostic observation schedule. Los Angeles, CA: Western Psychological Services.

Markova, I. S., \& Berrios, G. E. (2011). Awareness and insight in psychopathology: An essential distinction? Theory and Psychology, 21(4), 421-437. doi:10.1177/0959354310375330.

Owens, J. S., Goldfine, M. E., Evangelista, N. M., Hoza, B., \& Kaiser, N. M. (2007). A critical review of self-perceptions and the positive illusory bias in children with ADHD. Clinical Child and Family Psychological Review, 10, 335-351. doi:10.1007/s 10567-007-0027-3.

Quee, P. J., van der Meer, L., Bruggeman, R., de Haan, L., Krabbendam, L., Cahn, W., et al. (2011). Insight in psychosis: Relationship with neurocognition, social cognition and clinical symptoms depends on phase of illness. Schizophrenia Bulletin, 37, 29-37.

Roge, B., \& Mullet, E. (2011). Blame and forgiveness judgments among children, adolescents and adults with autism. Autism, 1-10. doi:10.1177/1362361310394219.

Russell, E., \& Sofronoff, K. (2005). Anxiety and social worries in children with Asperger Syndrome. Australian and New Zealand Journal of Psychiatry, 39(7), 633-638.

Saeedi, H., Addington, J., \& Addington, D. (2007). The association of insight with psychotic symptoms, depression, and cognition in early psychosis: A 3-year follow-up. Schizophrenia Research, 89, 123-128. doi:10.1016/j.schres.2006.09.018.

Sapara, A., Cooke, M., Fannon, D., Francis, A., Buchanan, R. W., Anikumar, A. P., et al. (2007). Prefrontal cortex and insight in schizophrenia: A volumetric MRI study. Schizophrenia Research, 89, 22-34. doi:S0920-9964(06)00413-0.

Schwartz, R. C., Cohen, B. N., \& Grubaugh, A. (1997). Does insight affect long-term impatient treatment outcome in chronic schizophrenia? Comprehensive Psychiatry, 38(5), 283-288.

Takeda, T., Kasia, K., \& Kato, N. (2007). Moral judgement in highfunctioning pervasive developmental disorders. Psychiatry and Clinical Neuroscience, 61, 407-414. doi:10.1111//j.1440-1819. 2007.01678.x.

Taylor, S. E., \& Brown, J. D. (1988). Illusions and well-being: A social psychological perspective on mental health. Psychological Bulletin, 103, 193-210.

Wechsler, D. (1999). Wechsler abbreviated scale of intelligence (WASI). San Antonio, TX: Harcourt Assessment. 